\title{
Next steps in Ewing sarcoma (epi-) genomics
}
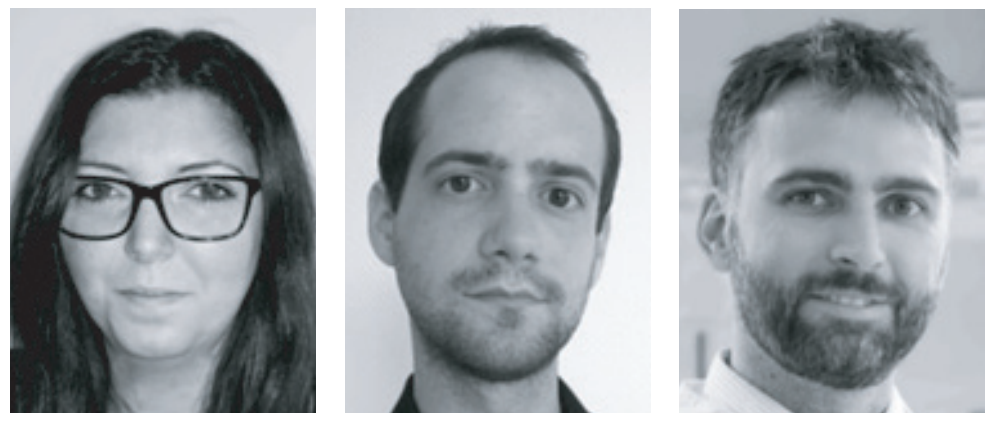

Giuseppina Sannino ${ }^{\ddagger, 1}$, Martin F Orth ${ }^{\ddagger, 1}$ \& Thomas GP Grünewald ${ }^{* \neq, 1}$

First draft submitted: 31 March 2017; Accepted for publication: 4 April 2017; Published online: 7 June 2017

Ewing sarcoma (EwS) is a highly aggressive bone-associated malignancy mostly affecting Caucasians. Recent studies illuminated its genetic and epigenetic architecture holding promise for clinical exploitation. Here, we summarize key findings and propose next steps for future research toward personalized medicine.

\section{Somatic mutations as drivers \&} biomarkers for EwS progression

EwS was the first solid tumor identified, caused by a chimeric fusion oncogene. The most common $(85 \%)$ translocation $\mathrm{t}(11 ; 22)$ (q24;q12) in EwS fuses EWSR1 with the E26 transformation-specific (ETS) DNA binding domain of FLII [1]. The second most frequent translocation $(10 \%) \mathrm{t}(21 ; 22)$ (q22; q12) fuses EWSRI with the ETSfamily member $E R G$ [2]. Although $E W S R I$ can fuse with different ETS members, and despite different fusion types with variable numbers of retained exons exist, this appears irrelevant for the EWSR1-ETS function and has no impact on patient outcome [3].
In 2014, three major sequencing studies proved that EwS is characterized by a striking paucity of additional somatic mutations [4-6]. Besides EWSR1-ETS fusions, the second most common mutations are found in STAG2 ( $15-20 \%$ of the cases) [4-6]. Although these inactivating mutations tended to be more frequent in metastatic disease $[4,5]$, they only affected outcome when co-occurring with TP53mutations [6]. Interestingly, while STAG2mutations were commonly only detected in primary tumors at subclonal levels, they were present at clonal levels at relapse. This suggests that STAG2-mutations confer a growth advantage and/or resistance to therapy, which may explain its high frequency $(\sim 40 \%)$ in EwS cell lines [4-6]. Functionally, STAG2 regulates sister chromatin separation during cell division as part of the cohesin complex and inactivating the mutations cause loss of genomic integrity in many cancers [7]. Accordingly, more copy number variations were found in EwS with STAG2-mutation [6].

\section{KEYWORDS}

- epigenetics • Ewing sarcoma

- EWSR1-FLI1 • genomics • STAG2

“...Ewing sarcoma is characterized by a striking paucity of additional somatic mutations.” 
Copy number variations in EwS mostly concern chrlq-gains, trisomy of chr8 and chr12, as well as losses of chr9p21 (comprising the $C D K N 2 A$ locus) and chr16q [6,8]. Chrlq-gains (18-31\% of the cases) correlate with metastasis and significantly worse outcome $[6,8,9]$, possibly through upregulation of CDT2, which promotes a highly proliferative phenotype [9]. Similarly, loss of chr16q is associated with worse outcome $[6,8]$. While one study found a mutual exclusivity of STAG2-mutation and loss of CDKN2A [6], two others did not $[4,5]$.

Next steps should include a transcriptomic comparison of STAG2 mutated and nonmutated EwS to identify dysregulated genes that could be used for targeted therapy. Also, deep sequencing of matched pairs of primary and relapse samples will be required to determine the STAG2mutation frequency at subclonal levels and to clarify whether this contributes to disease progression.

\section{Germline genetics in EwS development $\&$ therapeutic response}

Several studies suggest that germline variants are associated with the onset of EwS [10-14]. Indeed, approximately $13 \%$ of the patients carry likely pathogenic germline mutations in DNA repair genes, rendering genetic counselling essential [11]. While a specific single nucleotide polymorphism in EWSR1 was reported to predispose to EwS [14], possibly via favoring breakage events and thus, formation of EWSR1-ETS, a genomewide association study yielded no confirmative evidence [13]. Yet, specific features make EwS a genuine model for studying how germline variants and somatic mutations cooperate to promote tumorigenesis, tumor progression and therapeutic response [15]:

- EwS features a simple genetic make-up with a nearly diploid genome, a single somatic driver mutation (EWSR1-FLI1) [16] and only a few germline susceptibility loci [13].

- EWSR1-FLI1 can bind as a pioneer transcription factor to otherwise nonfunctional GGAA-microsatellites (mSats), thereby converting them into potent enhancers $[17,18]$. As, most GGAA-mSats underlie strong germline variability, they may contribute in concert with EWSR1-FLI1 to the variable incidence of EwS in different populations and to heterogeneous expression of relevant EWSR1-FLI1 target genes [15].
In line with this concept, several groups found that caucasians have more continuous GGAArepeats at specific EWSR1-FLI1-bound GGAAmSats, potentiating EWSR1-FLI1-induced expression of downstream oncogenes [19-21]. As an underlying mechanism, it was proposed that EWSR1-FLI1 clustering at GGAA-mSats leads to polymerization of the low-complexity domains of EWSR1 [22], which may constitute a scaffold for RNA polymerase II [22]. This would provide a rationale for the positive correlation of the number of continuous GGAA-repeats with the enhancer activity of EWSR1-FLI1-bound GGAA-mSats [23]. As specific genes driven through the oncogenic interplay of EWSR1FLI1 and GGAA-mSats may partially explain the apparent heterogeneity in clinical outcome, next steps should improve our understanding of how these genes are activated and how to translate this knowledge into novel therapies [15].

Besides GGAA-mSats, also germline variation in the exome may contribute to the clinical phenotypes in EwS. For instance, single nucleotide polymorphisms in the EWSR1-FLI1 target CD99 [24] as well as in pharmacokinetic genes such as $A B C C 6, A B C B 1$ and $C Y P 2 C 8$ seem to correlate with the clinical outcome of EwS patients [25]. As these three pharmacokinetic genes are virtually not expressed in EwS tumors (own analyses of publicly available microarray data; GSE34620 [13]), the underlying mechanism causing this statistical association remains elusive. More plausibly, another study reported on a rare coding variant in $R A R G$ - a gene highly expressed in heart - which alters its function and is associated with anthracycline-induced cardiotoxicity in several pediatric malignancies including EwS [26].

Next steps should include similar analyses of genetically-matched $\mathrm{EwS}$ patients, and probe on a genome-wide level the contribution of germline variation to drug responses for all drugs (and related toxicities) employed in the current therapy protocols.

\section{Epigenomics as a potential key for the oncogenic effects of EWSR1-ETS}

Since the germline variants and few somatic mutations cannot fully explain the apparent clinical heterogeneity in EwS yet, epigenetic alterations in conjunction with EWSR1-FLI1 were comprehensively analyzed $[27,28]$. The preferred DNAbinding motifs of EWSR1-ETS are known since several years. In chromatin immunoprecipitation 
and next-generation sequencing (ChIP-Seq) for EWSR1-FLI1 evenly spread peaks appear across the genome with enrichment at GGAAmSats upstream of EWSR1-FLI1 upregulated genes [29,30]. However, it became evident that the DNA-binding of EWSR1-FLI1 depends on DNA accessibility and vice versa, that EWSR1-FLI1 strongly impacts the epigenome. Indeed, EWSR1-FLI1 ChIP-Seq peaks close to EWSR1-FLI1 target genes are flanked by histone marks typical for the enhancers $[18,28,30,31]$, bound by RNA polymerase II [31] and depleted for repressive marks [28,31]. In contrast, GGAA$\mathrm{mSats}$ in normal human cells feature repressive epigenomic signatures [31].

The strong regulatory role of EWSR1-FLI1 in epigenomics is documented by the fact that its expression in HUVEC cells leads to nucleosome depletion, while its knockdown in EwS cells restores nucleosome occupancy [31]. Nucleosome depletion has been reported at the same GGAA$\mathrm{mSats}$ that exhibit histone acetylation in mesenchymal stem cells (MSCs), which results in more accessible chromatin. Hence, EWSR1-FLI1 likely hijacks the open chromatin structure of MSCs, to eventually induce total nucleosome loss and unfold its oncogenic potential. This lends further support for MSCs representing cells of origin of EwS [32].

Deregulation of EWSR1-FLI1 primarily affects the active enhancer mark H3K27ac [18,28], which is enriched at the promoters and enhancers of EWSR1-FLI1 upregulated genes, but depleted at the genes being negatively correlated with EWSR1-FLI1 expression [28]. H3K27ac peaks, usually, strongly overlap with superenhancers and, consistently, most superenhancers in EwS show EWSR1-FLI1-binding and H3K27ac modification [28]. Conversely, after downregulation of EWSR1-FLI1 in EwS cells, the chromatin structure resembles that of nontransformed MSCs with major changes in H3K27ac [18].

As EWSR1-FLI1 cannot directly modify histones, the effect on H3K27ac must be mediated via other proteins $[31,33]$. In accordance, EWSR1FLI1 multimers at GGAA-mSats bind to p300 - an acetyltransferase regulating chromatin and enhancer activity - thereby guiding it to regulatory elements of EWSR1-FLI1 upregulated genes [18]. At EWSR1-FLI1 repressed genes, one single EWSR1-FLI1 binds to canonical ETS motifs, displacing the normal ETS transcription factors [18]. In contrast to the wildtype ETS transcription factors, EWSR1-FLI1 lacks one of the two $\mathrm{p} 300$ binding domains. Thus, monomeric EWSR1-FLI1 does not recruit p300 to the repressed sites, leading to lower H3K27ac [18]. This can partially explain why EWSR1-FLI1 downregulates approximately as many genes as it upregulates $[18,28,29]$ and is compatible with the hypothesis of RNA polymerase II-binding by EWSR1-FLI1 polymers [22]. Furthermore, EWSR1-FLI1 binds two components of the MLL family protein complexes, which catalyze H3K4 methylation [18]. Collectively, EWSR1FLI1 establishes cell-type specific (super-) enhancers by recruiting histone acetyl- and methyltransferases $[18,28]$.

The modulation of chromatin structure by EWSR1-FLI1 is clinically relevant: histone deacetylase inhibitors, normally favoring open chromatin structure, surprisingly reverse the epigenomic signature induced by EWSR1-FLI1 and upregulate otherwise downregulated genes [33], possibly by EWSR1-FLI1 transcription modulation. Next steps should address the underlying mechanisms, the efficacy of other chromatin modulating therapeutics, and whether the therapeutic effect of histone deacetylase inhibitors exclusively relies on downregulation of EWSR1-FLI1 [33].

Recent data confirmed that EWSR1-FLI1bound enhancers are hypomethylated [27]. Sheffield $e t$ al. also reported on a EwS-specific epigenomic signature and a continuous intertumoral spectrum of DNA methylation, which likely represent the differentiation state of the cell of origin and its reprogramming by EWSR1-FLI1 [27]. Interestingly, a more stem cell-like methylation signature correlated positively with STAG2-mutation, whereas a more EwS-specific signature correlated with TP53mutation [27]. Since DNA methylation is highly heterogeneous within each tumor and as higher epigenetic intratumor heterogeneity appeared to correlate with metastasis [27], this might be partially attributable to subclonal STAG2mutations. Next steps should aim at increasing our understanding of the modulatory effects of EWSR1-FLI1 on chromatin structure and of interindividual differences in epigenomics, which may improve personalized therapeutic protocols.

\section{Financial \& competing interests disclosure}

The laboratory of TGP Grünewald is supported by grants

from the 'Verein zur Förderung von Wissenschaft und “...most super-enhancers in Ewing sarcoma show EWSR1-FLI1-binding and H3K27ac modification.” 
Forschung an der Medizinischen Fakultät der LMU München (WiFoMed)', by LMU Munich's Institutional Strategy LMUexcellent within the framework of the German Excellence Initiative, the 'Mehr LEBEN für krebskranke Kinder - Bettina-Bräu-Stiftung', the Walter Schulz Foundation, the Friedrich-Baur Foundation, the Fritz-Thyssen-Foundation (FTF-2015-01046 to GSannino), the Wilhelm Sander-Foundation (2016.167.1) and by the German Cancer Aid (DKH-111886 and DKH70112257). The authors have no other relevant affiliations or financial involvement with any organization or entity with a financial interest in or financial conflict with the subject matter or materials discussed in the manuscript apart from those disclosed.

No writing assistance was utilized in the production of this manuscript.

\section{References}

1 Delattre O, Zucman J, Plougastel B et al. Gene fusion with an ETS DNA-binding domain caused by chromosome translocation in human tumours. Nature 359(6391), 162-165 (1992).

2 Sorensen PH, Lessnick SL, Lopez-Terrada D, Liu XF, Triche TJ, Denny CT. A second Ewing's sarcoma translocation, $t(21 ; 22)$, fuses the EWS gene to another ETS-family transcription factor, ERG. Nat. Genet. 6(2), 146-151 (1994).

3 Le Deley M-C, Delattre O, Schaefer K-L et al. Impact of EWS-ETS fusion type on disease progression in Ewing's sarcoma/peripheral primitive neuroectodermal tumor: prospective results from the cooperative Euro-E.W.I.N.G. 99 trial. J. Clin. Oncol. 28(12), 1982-1988 (2010).

4 Brohl AS, Solomon DA, Chang W et al. The genomic landscape of the Ewing Sarcoma family of tumors reveals recurrent STAG2 mutation. PLoS Genet. 10(7), e1004475 (2014).

5 Crompton BD, Stewart C, Taylor-Weiner A et al. The genomic landscape of pediatric Ewing sarcoma. Cancer Discov. 4(11), 1326-1341 (2014).

6 Tirode F, Surdez D, Ma X et al. Genomic landscape of Ewing sarcoma defines an aggressive subtype with co-association of STAG2 and TP53 mutations. Cancer Discov. 4(11), 1342-1353 (2014).

7 Solomon DA, Kim T, Diaz-Martinez LA et al. Mutational inactivation of STAG2 causes aneuploidy in human cancer. Science 333(6045), 1039-1043 (2011).

8 Brisset S, Schleiermacher G, Peter M et al. $\mathrm{CGH}$ analysis of secondary genetic changes in Ewing tumors: correlation with metastatic disease in a series of 43 cases. Cancer Genet. Cytogenet. 130(1), 57-61 (2001).

9 Mackintosh C, Ordóñez JL, GarcíaDomínguez DJ et al. 1q gain and CDT2 overexpression underlie an aggressive and highly proliferative form of Ewing sarcoma. Oncogene 31(10), 1287-1298 (2012).
10 Ballinger ML, Goode DL, Ray-Coquard I et al. Monogenic and polygenic determinants of sarcoma risk: an international genetic study. Lancet Oncol. 17(9), 1261-1271 (2016).

11 Brohl AS, Patidar R, Turner CE et al. Frequent inactivating germline mutations in DNA repair genes in patients with Ewing sarcoma. Genet. Med. doi:10.1038/ gim.2016.206 (2017) (Epub ahead of print).

12 DuBois SG, Goldsby R, Segal M et al. Evaluation of polymorphisms in EWSR1 and risk of Ewing sarcoma: a report from the Childhood Cancer Survivor Study. Pediatr. Blood Cancer 59(1), 52-56 (2012).

13 Postel-Vinay S, Véron AS, Tirode F et al. Common variants near TARDBP and EGR2 are associated with susceptibility to Ewing sarcoma. Nat. Genet. 44(3), 323-327 (2012).

14 Silva DSBS, Sawitzki FR, De Toni EC et al. Ewing's sarcoma: analysis of single nucleotide polymorphism in the EWS gene. Gene 509(2), 263-266 (2012).

15 Grünewald TGP, Delattre O. Cooperation between somatic mutations and germline susceptibility variants in tumorigenesis - a dangerous liaison. Mol. Cell. Oncol. 3(3), e1086853 (2016).

16 Sand LGL, Szuhai K, Hogendoorn PCW. Sequencing overview of Ewing sarcoma: a journey across genomic, epigenomic and transcriptomic landscapes. Int. J. Mol. Sci. 16(7), 16176-16215 (2015).

17 Gangwal K, Sankar S, Hollenhorst PC et al. Microsatellites as EWS/FLI response elements in Ewing's sarcoma. Proc. Natl Acad. Sci. USA 105(29), 10149-10154 (2008).

18 Riggi N, Knoechel B, Gillespie SM et al. EWS-FLI1 utilizes divergent chromatin remodeling mechanisms to directly activate or repress enhancer elements in Ewing sarcoma. Cancer Cell 26(5), 668-681 (2014).

19 Beck R, Monument MJ, Watkins WS et al. EWS/FLI-responsive GGAA microsatellites exhibit polymorphic differences between European and African populations. Cancer Genet. 205(6), 304-312 (2012).
20 Grünewald TGP, Bernard V, GilardiHebenstreit P et al. Chimeric EWSR1-FLI1 regulates the Ewing sarcoma susceptibility gene EGR2 via a GGAA microsatellite. Nat. Genet. 47(9), 1073-1078 (2015).

21 Monument MJ, Johnson KM, McIlvaine E et al. Clinical and biochemical function of polymorphic NR0B1 GGAA-microsatellites in Ewing sarcoma: a report from the Children's Oncology Group. PLoS ONE 9(8), e104378 (2014).

22 Kwon I, Kato M, Xiang $S$ et al. Phosphorylation-regulated binding of RNA polymerase II to fibrous polymers of low-complexity domains. Cell 155(5), 1049-1060 (2013).

23 Gangwal K, Lessnick SL. Microsatellites are EWS/FLI response elements: genomic "junk" is EWS/FLI's treasure. Cell Cycle 7(20), 3127-3132 (2008).

24 Martinelli M, Parra A, Scapoli L et al. CD99 polymorphisms significantly influence the probability to develop Ewing sarcoma in earlier age and patient disease progression. Oncotarget 7(47), 77958-77967 (2016).

25 Ruiz-Pinto S, Pita G, Patiño-García A et al. Identification of genetic variants in pharmacokinetic genes associated with Ewing Sarcoma treatment outcome. Ann. Oncol. 27(9), 1788-1793 (2016).

26 Aminkeng F, Bhavsar AP, Visscher $\mathrm{H}$ et al. A coding variant in RARG confers susceptibility to anthracycline-induced cardiotoxicity in childhood cancer. Nat. Genet. 47(9), 1079-1084 (2015).

27 Sheffield NC, Pierron G, Klughammer J et al. DNA methylation heterogeneity defines a disease spectrum in Ewing sarcoma. Nat. Med. 23(3), 386-395 (2017).

28 Tomazou EM, Sheffield NC, Schmidl C et al. Epigenome mapping reveals distinct modes of gene regulation and widespread enhancer reprogramming by the oncogenic fusion protein EWS-FLI1. Cell Rep. 10(7), 1082-1095 (2015). 
29 Bilke S, Schwentner R, Yang F et al. Oncogenic ETS fusions deregulate E2F3 target genes in Ewing sarcoma and prostate cancer. Genome Res. 23(11), 1797-1809 (2013).

30 Wei G-H, Badis G, Berger MF et al. Genome-wide analysis of ETS-family DNA-binding in vitro and in vivo. EMBO J. 29(13), 2147-2160 (2010).
31 Patel M, Simon JM, Iglesia MD et al. Tumorspecific retargeting of an oncogenic transcription factor chimera results in dysregulation of chromatin and transcription. Genome Res. 22(2), 259-270 (2012).

32 Gomez NC, Hepperla AJ, Dumitru R, Simon JM, Fang F, Davis IJ. Widespread chromatin accessibility at repetitive elements links stem cells with human cancer. Cell Rep. 17(6), 1607-1620 (2016).

33 Pattenden SG, Simon JM, Wali A et al. High-throughput small molecule screen identifies inhibitors of aberrant chromatin accessibility. Proc. Natl Acad. Sci. USA 113(11), 3018-3023 (2016). 